\title{
PROGRESS IN SOLAR RADIO IMAGING WITH THE UTR-2 RADIO TELESCOPE AT DECAMETER WAVELENGTHS
}

\author{
A. A. Stanislavsky*, A. A. Konovalenko*, A. A. Koval ${ }^{* \dagger}$, \\ E. P. Abranin*, Ya. S. Volvach*, and L. A. Stanislavsky ${ }^{\ddagger}$
}

\begin{abstract}
The newly modernized analog-digital heliograph, based on the UTR-2 radio telescope, builds two-dimensional pictures of the outer solar corona in the frequency range of $8-32 \mathrm{MHz}$ with a frequency resolution of $4 \mathrm{kHz}$ and a time resolution up to $1 \mathrm{~ms}$, having a dynamic range of about $90 \mathrm{~dB}$. The instrument was originally put into operation in the late seventies of the last century, and the radio astronomy observations with the UTR-2 array were made only near to several fixed frequencies, while the antenna system capabilities in frequency broadbandness were much wider. The modern advance of computer electronics served as an impetus to the development of this tool. The heliograph has been upgraded significantly. It has a hybrid scheme that combines the best features of both digital and analog technologies at a relatively low price. This device works on the parallel-series principle by using five equi-spaced array pattern beams which scan the solar corona at a predetermined rate. Consequently, each solar image presents a frame of $5 \times 8$ pixels with the spatial resolution of $25^{\prime} \times 25^{\prime}$ at $25 \mathrm{MHz}$. Each pixel reveals the signal level received with the corresponding antenna pattern beam by the digital receiver/spectrometer in real time. Starting with a brief overview of the heliograph design, we outline potential capabilities of this instrument in radio imaging from recent observations.
\end{abstract}

* Institute of Radio Astronomy, National Academy of Sciences of the Ukraine, Kharkiv, Ukraine

${ }^{\dagger}$ Institute of Space Sciences and School of Space Science and Physics, Shandong University, Weihai, China

${ }^{\ddagger}$ Faculty of Radio Systems for Aircraft, National Aerospace University "KhAI", Kharkiv, Ukraine 
